\title{
Rifabutin-Containing Triple Therapy (RHB-105) for Eradication of Helicobacter pylori: Randomized ERADICATE Hp Trial
}

\author{
Ira N. Kalfus ${ }^{1, *}$, David Y. Graham ${ }^{2}$, Dennis S. Riff ${ }^{3}$ and Raymond M. Panas ${ }^{4}$ \\ 1 Independent Consultant, M2g Consulting, 251 Central Park West, New York, NY 10024, USA \\ 2 Department of Medicine, Michael E. DeBakey VA Medical Center and Baylor College of Medicine, \\ 2002 Holcombe Boulevard, Houston, TX 77030, USA; dgraham@bcm.edu \\ 3 Anaheim Clinical Trials, 1085 N. Harbor Blvd, Anaheim, CA 92801, USA; dsriff@aol.com \\ 4 Medical Affairs, RedHill Biopharma, Inc., 8045 Arco Corporate Drive, Suite 200, Raleigh, NC 27617, USA; \\ raypanas@gmail.com \\ * Correspondence: ikalfus@aol.com; Tel.: +19-(17)-8177517
}

Received: 10 September 2020; Accepted: 5 October 2020; Published: 9 October 2020

\begin{abstract}
Due to increasing resistance to commonly used antibiotics, the World Health Organization and Food and Drug Administration have advocated the development of new therapeutic regimens for Helicobacter pylori (H. pylori). This phase three, double-blind study (ERADICATE Hp) randomized (2:1) treatment-naïve adults with $H$. pylori infection and dyspepsia to RHB-105 (an all-in-one combination of omeprazole $40 \mathrm{mg}$, amoxicillin $1000 \mathrm{mg}$, and rifabutin $50 \mathrm{mg}$ ) or an identically-appearing placebo, both administered every $8 \mathrm{~h}$ for 14 days. The H. pylori eradication rate with RHB-105, using a modified intent-to-treat (mITT) population of subjects who received $\geq 1$ dose of study drug and had test-of-eradication performed 28-35 days post-completion of therapy, was compared (one-sample Z-test) to a literature-derived comparator rate of $70 \%$ and success rate with physician-selected standard-of-care given to placebo failures. The mITT H. pylori eradication rate (95\% CI) with RHB-105 of $89.4 \%(82.0-96.8 \%)$ was greater than both the literature-derived comparator rate $(P<0.001)$ and the standard-of-care rate of $63.0 \%(44.8-81.1 \%)(P=0.006)$. Adverse events with an incidence $\geq 5 \%$ for RHB-105 were diarrhea (12.7\%), headache (11.9\%), chromaturia (9.3\%), abdominal tenderness $(6.8 \%)$, and dizziness (5.1\%). No leukopenia was noted. RHB-105 (Talicia ${ }^{\circledR}$ ) proved to be a safe and effective empiric therapy for $H$. pylori eradication.
\end{abstract}

Keywords: RHB-105; rifabutin; Helicobacter pylori; standard-of-care; dyspepsia; clinical trial

\section{Introduction}

Helicobacter pylori (H. pylori) is recognized as the primary etiologic agent responsible for gastritis, peptic ulcers, and gastric cancer [1,2]. Importantly, $H$. pylori is classified as a Group 1 carcinogen by the International Agency for Research on Cancer [3] and is considered the primary cause of human gastric cancer. A recent analysis of over 370,000 health records from the Veteran's Administration database showed that eradication of $H$. pylori from patients with confirmed infection resulted in a $75 \%$ reduction in the risk of developing gastric cancer [4]. The World Health Organization (WHO) estimates that $H$. pylori is present in about half of the world's population, while the Centers for Disease Control and Prevention (CDC) estimates that 30-40\% of Americans harbor the infection [5-8]. Specifically, in the United States, the prevalence by race is higher in Hispanics (60\%) and African Americans (54\%) compared to White non-Hispanics (20\%) [9]. While there is a similarity in infection rates by gender, age over 60 years presents a higher risk of infection (50\%) compared to those under 30 years (20\%) [9]. 
The clinical utility of current treatment guidelines for eradication of $H$. pylori infections has steadily declined due to the growing resistance to commonly prescribed antibiotic therapies $[10,11]$. H. pylori antibiotic resistance, particularly to clarithromycin, metronidazole, and levofloxacin, is now a major concern among clinicians [1,11-13]. This recent decline in therapeutic efficacy is the key rationale for why the WHO and United States (US) Food and Drug Administration (FDA) is targeting H. pylori for the development of new therapeutic agents $[10,14]$. Typical regimens of treatment currently include triple therapy (e.g., a proton pump inhibitor (PPI) with amoxicillin and a second antibiotic) or a quadruple therapy (e.g., PPI with bismuth and two antibiotics) with treatment durations ranging from $7-14$ days $[1,12,13]$. However, the effect of the worldwide increase in antimicrobial resistance, including those antibiotics empirically used to treat $H$. pylori infection, has led to calls for new antibiotic regimens to eradicate $H$. pylori.

The rifamycin derivative rifabutin is potentially an excellent option for $H$. pylori eradication as rifabutin (1) provides bactericidal activity against $H$. pylori [15-20], (2) achieves high intracellular and intragastric concentrations [21,22], and (3) resistance is rare even after failure to cure H. pylori [23]. Rifabutin resistance has generally been reported only after the administration of high doses for extended durations such as those required for the treatment of mycobacterial infections. Prior experience has shown that the risk of development of antimicrobial resistance with $H$. pylori is further reduced when it is used with another antimicrobial agent such as amoxicillin [24,25]. In addition, the development of rifabutin resistant to H. pylori in vitro has shown to be very low (estimated at one in $10^{9}$ ) [17].

A prior meta-analysis of 2982 subjects receiving rifabutin triple therapy for H. pylori eradication reported a mean eradication rate of $73 \%$ (range of $66-79 \%$ ) despite one to four prior treatment failures with other regimens [23]. The recent American College of Gastroenterology (ACG) Clinical Guideline for the treatment of $H$. pylori infection also recommends high dose rifabutin (300 mg once daily dose) as salvage therapy for those who fail multiple courses of $H$. pylori therapy [11]. However, Borody et al. studied a lower dose rifabutin regimen that provided high cure rates in 130 subjects who had failed standard clarithromycin-based triple therapy [26]. They achieved an overall eradication rate of $90.8 \%$, and resistance to metronidazole or clarithromycin did not reduce the $H$. pylori eradication rate [26]. Based on this experience, RedHill Biopharma designed and implemented the ERADICATE Hp study (NCT01980095) to assess the safety and efficacy of RHB-105, an all-in-one capsule containing a fixed-dose combination of omeprazole (120 mg/day), amoxicillin ( $3000 \mathrm{mg} /$ day), and rifabutin (150 mg/day) in patients with proven $H$. pylori infections. Results of the double-blind, placebo-controlled ERADICATE Hp study demonstrate that RHB-105, an all-in-one rifabutin triple therapy, is a safe and effective empiric therapy for H. pylori eradication.

\section{Study Results}

\subsection{Participants}

Overall, 277 subjects were screened, 119 subjects were randomized, and 118 subjects received at least one dose of study drug (77 and 41 in the RHB-105 and placebo groups, respectively) (Figure 1). The treatment groups were similar, in general, based on demographics (Table 1). Subjects were primarily female $(62.7 \%)$ and White $(92.4 \%)$; their average age was 46.0 years. More subjects in the placebo group were Hispanic ( $85.4 \%$ vs. $77.9 \%$ of subjects in the RHB-105 group). Ninety-three subjects $(78.8 \%)$ completed the study, and $25(21.2 \%)$ discontinued prematurely. 


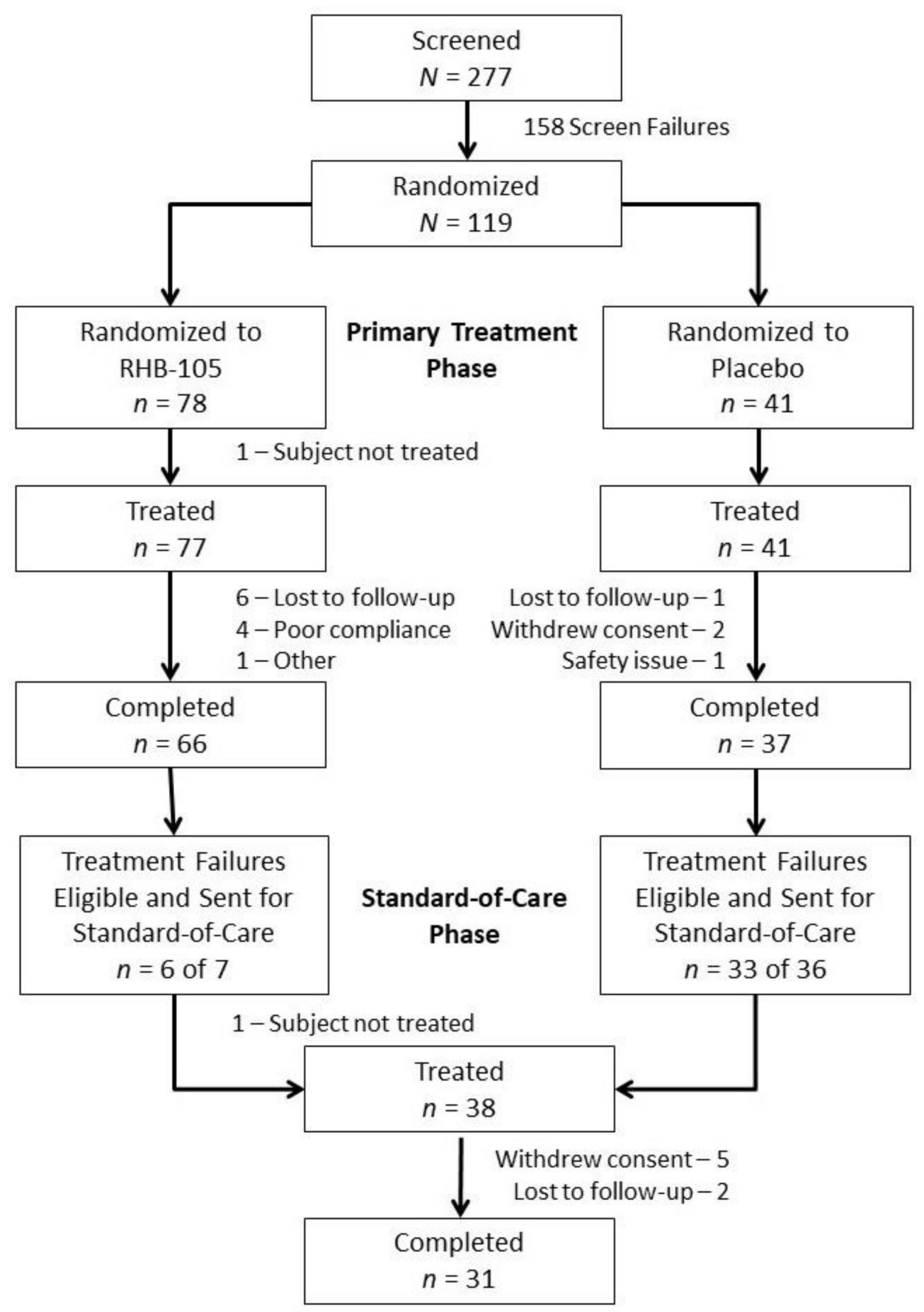

Figure 1. Consolidated Standards of Reporting Trials (CONSORT) Diagram.

Forty-three treatment failures ( 7 and 36 subjects in the RHB-105 and placebo groups, respectively) were eligible to be treated in the standard-of-care phase (Figure 1); 39 subjects chose to participate, and 38 received physician-selected standard-of-care. Three of the seven treatment failures in the RHB-105 group agreed to undergo endoscopy; all three strains of $H$. pylori isolated were susceptible to amoxicillin and clarithromycin, and two of three were susceptible to metronidazole. During this second phase of the study, 31 subjects completed standard-of-care treatment and underwent follow-up test-of-eradication to determine $H$. pylori eradication status. 
Table 1. Baseline subject demographics and characteristics.

\begin{tabular}{cccc}
\hline Characteristic & RHB-105 $\boldsymbol{n = 7 7}$ & Placebo $\boldsymbol{n = 4 1}$ & Total $\mathbf{N}=\mathbf{1 1 8}$ \\
\hline Age, $\mathrm{y}$, mean \pm SD & $46.2 \pm 10.56$ & $45.8 \pm 9.52$ & $46.0 \pm 10.18$ \\
\hline Sex, $\mathrm{n}(\%)$ & & & \\
Female & $49(63.6 \%)$ & $25(61.0 \%)$ & $74(62.7 \%)$ \\
Male & $28(36.4 \%)$ & $16(39.0 \%)$ & $44(37.3 \%)$ \\
\hline Ethnicity, n (\%) & & \\
Hispanic or Latino & $60(77.9 \%)$ & $35(85.4 \%)$ & $95(80.5 \%)$ \\
Not Hispanic or Latino & $17(22.1 \%)$ & $6(14.6 \%)$ & $23(19.5 \%)$ \\
\hline Race, $\mathrm{n}(\%)$ & & & $109(92.4 \%)$ \\
White & $71(92.2 \%)$ & $38(92.7 \%)$ & $9(7.6 \%)$ \\
\hline
\end{tabular}

\subsection{Treatment Efficacy Results}

For the primary endpoint (modified intent-to-treat (mITT)), the H. pylori eradication rate $(95 \% \mathrm{CI})$ with RHB-105 was $89.4 \%(82.0-96.8 \%)$ (Table 2$)$, which was significantly greater $(P<0.001)$ than the literature-derived historical comparator rate of $70 \%$. The $H$. pylori eradication rate was statistically significant $(P<0.001)$ for all sites (or cluster of sites that enrolled four or fewer subjects) with the exception of two sites $(66.7 \%(n=3), P=0.549 ; 81.8 \%(n=22), P=0.075)$. In a sensitivity analysis based on the intent-to-treat (ITT) population, the between-group difference (H. pylori eradication rate of $76.6 \%$ (vs. $70 \%$; $P=0.085$ )) did not reach the level of statistical significance which was greatly influenced by 11 subjects failing to return for ${ }^{13} \mathrm{C}$ urea breath test (UBT) at the test-of-eradication visit. The results of the sensitivity analysis in the per-protocol (PP) population (H. pylori eradication rate of $88.9 \%$ (vs. $70 \% ; P<0.001)$ ) was similar to the primary analysis. Among the placebo subjects, one subject was noted to be $H$. pylori negative at the test-of-eradication visit (eradication rate: $2.4 \%$ (1/41)—ITT; $2.7 \%(1 / 37) —$ mITT; $2.8 \%$ (1/36)—PP). While the H. pylori eradication rate with RHB-105 was significantly greater compared to that of placebo $(P<0.001)$, this evaluation was not considered relevant or informative for efficacy.

Table 2. Helicobacter pylori eradication rate: RHB-105 compared to literature-derived historical control and physician-selected standard-of-care.

\begin{tabular}{cccc}
\hline & ITT $^{\text {a }}$ & mITT & PP \\
\hline RHB-105 & $\mathbf{7 6 . 6 \% ( 5 9 / 7 7 )}$ & $\mathbf{8 9 . 4 \% ( 5 9 / 6 6 )}$ & $\mathbf{8 8 . 9 \% ( 5 6 / 6 3 )}$ \\
95\% CI & $67.2-86.1 \%$ & $82.0-96.8 \%$ & $81.1-96.7 \%$ \\
Literature-derived Historical Control ${ }^{\mathrm{b}}$ & $70 \%$ & $70 \%$ & $70 \%$ \\
$\begin{array}{c}\text {-value, RHB-105 vs. Literature-derived } \\
\text { Historical Control }\end{array}$ & 0.085 & $<0.001$ & $<0.001$ \\
\hline $\begin{array}{c}\text { Physician-selected Standard-of-Care } \\
\text { (Placebo Subjects) } \\
\text { 95\% CI }\end{array}$ & $51.5 \%(17 / 33)$ & $63.0 \%(17 / 27)$ & NA \\
$\begin{array}{c}P \text {-value, RHB-105 vs. Physician-selected } \\
\text { Standard-of-Care }\end{array}$ & $34.5-68.6 \%$ & $44.8-81.1 \%$ & \\
\hline
\end{tabular}

ITT = intent-to-treat; mITT = modified intent-to-treat; NA = not applicable; $\mathrm{PP}=$ per-protocol. ${ }^{\text {a }}$ Eleven of 77 subjects in the ITT population failed to return for test-of-eradication at visit four and were imputed as treatment failures. ${ }^{\mathrm{b}}$ Estimated Helicobacter pylori eradication rate of 70\% derived from references [27-29]. Notes: The ITT population included all subjects who received at least one dose of randomized study treatment; those who were lost to treatment, dropped out of the study, or failed to return for test-of-eradication were imputed as treatment failures. The mITT population included all subjects who received at least 1 dose of randomized study treatment and underwent ${ }^{13} \mathrm{C}$ urea breath test (UBT) test-of-eradication 28-35 days post-completion of therapy. The PP population included all subjects who consumed at least $75 \%$ of planned study treatment received, underwent ${ }^{13} \mathrm{C}$ UBT at the initial test-of-eradication visit, and did not have any major protocol violations. 


\subsection{CYP2C19 Genotyping}

In the mITT population, 65 subjects receiving RHB-105 had blood samples for CYP2C19 status tested. Based on CYP2C19 genotypes, there were 11 rapid, 38 normal, 16 intermediate, and no ultra-rapid or poor metabolizers. No statistically significant difference was observed $(P=0.123)$ between the H. pylori eradication and failure groups based on the CYP2C19 status (while assessing the distribution over the different genotypes) among subjects randomized to RHB-105.

\subsection{Physician-Selected Standard-of-Care Efficacy Results}

The H. pylori eradication rate with RHB-105 treatment during the double-blind phase was significantly greater than the rate following physician-selected standard-of-care of treatment-naïve placebo subjects (mITT: $89.4 \%$ vs $63.0 \%, P=0.006$; ITT: $76.6 \%$ vs $51.5 \%, P=0.009$; Table 2 ). While physicians could choose any standard-of-care therapy, a clarithromycin-containing triple regimen was the most commonly prescribed, for which the H. pylori eradication rate (mITT) was $60.9 \%$ (Table 3 ).

Table 3. H. pylori Eradication Rates with Physician-selected Standard-of-Care (mITT).

\begin{tabular}{cccc}
\hline & Placebo & RHB-105 Treatment Failures & Total \\
\hline All Subjects & $17 / 27(62.9 \%)$ & $2 / 4(50.0 \%)$ & $19 / 31(61.3 \%)$ \\
Clarithromycin triple therapy $^{\mathrm{a}}$ & $14 / 23(60.9 \%)$ & $2 / 4(50.0 \%)$ & $16 / 27(59.3 \%)$ \\
Metronidazole triple therapy $^{\mathrm{b}}$ & $1 / 2(50.0 \%)$ & 0 & $1 / 2(50.0 \%)$ \\
Bismuth quadruple therapy $^{\mathrm{c}}$ & $2 / 2(100.0 \%)$ & 0 & $2 / 2(100.0 \%)$ \\
\hline
\end{tabular}

mITT = modified intent-to-treat. ${ }^{a}$ Clarithromycin, amoxicillin, and proton pump inhibitor (omeprazole or lansoprazole). ${ }^{\mathrm{b}}$ Metronidazole, bismuth subcitrate potassium, and tetracycline. ${ }^{\mathrm{c}}$ Bismuth subcitrate potassium, metronidazole, tetracycline, and omeprazole.

\subsection{Safety Results}

Headache and diarrhea were the most commonly reported adverse events (Table 4). Chromaturia was also reported but is a known effect associated with the use of rifabutin. Most reported adverse events were mild or moderate in severity. A severe treatment-emergent adverse event was reported for four subjects: rash and diarrhea for one RHB-105-treated subject each and anemia and perirectal abscess for one placebo-treated subject each; the latter event was the only treatment-emergent serious adverse event reported. Three subjects discontinued due to adverse events. Among the RHB-105 subjects, one subject reported moderate pharyngitis, and one subject reported mild nausea with vomiting. Among the placebo subjects, one subject reported severe diarrhea with nausea and vomiting. Only the nausea with vomiting experienced by a RHB-105-treated subject was considered possibly related to the study drug. Bone marrow suppression, which has been reported with long term rifabutin use, was absent in this study.

Table 4. Adverse Events Occurring in $>2 \%$ of All Subjects and in a Greater Percentage of RHB-105 Subjects than Placebo Subjects.

\begin{tabular}{cccc}
\hline Adverse Event & RHB-105 $n=77$ & Placebo $\boldsymbol{n = 4 1}$ & Total $\mathbf{N = 1 1 8}$ \\
\hline All subjects with an adverse event & $39(50.6 \%)$ & $19(46.3 \%)$ & $58(49.2 \%)$ \\
\hline Headache a $^{\text {a }}$ Diarrhea & $12(15.6 \%)$ & $4(9.8 \%)$ & $16(13.6 \%)$ \\
Chromaturia & $11(14.3 \%)$ & $4(9.8 \%)$ & $15(12.7 \%)$ \\
Rash & $10(13.0 \%)$ & $1(2.4 \%)$ & $11(9.3 \%)$ \\
Nausea & $4(5.2 \%)$ & 0 & $4(5.2 \%)$ \\
Oropharyngeal pain & $3(3.9 \%)$ & $1(2.4 \%)$ & $4(3.4 \%)$ \\
Blood creatine phosphokinase increase & $3(3.9 \%)$ & 0 & $3(2.5 \%)$ \\
\hline
\end{tabular}

${ }^{\mathrm{a}}$ Headache includes headache and migraine. 


\section{Discussion}

The first approval of an H. pylori eradication therapy was nearly 25 years ago. No new therapies or combinations have been approved in the decade prior to the development of RHB-105. As such, there is a growing concern by agencies and organizations such as the WHO, FDA, ACG, and European Medicines Agency about the increasing lack of effective treatment options for $H$. pylori infection. Increasingly resistant $H$. pylori has been noted in the literature and confirmed by the findings from this study. Additionally, the general absence of knowledge about local resistance rates creates a challenge for physicians to treat $H$. pylori effectively and empirically. Together, this suggests the need for a new antibiotic treatment for eradicating $H$. pylori infections.

This double-blind, randomized, controlled trial investigated a rifabutin-containing triple therapy combining three drugs (rifabutin, amoxicillin, and omeprazole) into an all-in-one capsule. The H. pylori eradication rate with RHB-105 was statistically significantly greater than that achieved by the historical control in the protocol-defined mITT analysis. Pharmacogenetic testing suggested no significant findings based on the overall metabolism of RHB-105. The study also demonstrated the need for follow-up test-of-eradication assessment to verify eradication, as noted by the ITT population analysis. The evidence from this study supported the further development of RHB-105, which resulted in the conduct of a similar study that established rifabutin as a safe and effective first-line therapy for H. pylori infection [30]. Furthermore, among treatment failures given the option for physician-selected standard-of-care, clarithromycin-containing triple therapy was by far the first choice by most physicians, despite guidelines emphasizing issues regarding clarithromycin resistance [12,13]. RHB-105 was well-tolerated based upon comparison to placebo control, with diarrhea and headache being the most commonly reported adverse events. The results of EDADICATE-Hp and the companion phase three study EDADICATE-Hp2 [30] formed the basis for FDA approval of RHB-105 as first-line treatment for eradicating $H$. pylori.

Study limitations included potential for unblinding given chromaturia present with RHB-105. However, the hard endpoint of ${ }^{13} \mathrm{C}$ UBT mitigates this concern. Placebo was not an ideal comparator given the lack of efficacy with placebo; however, a placebo-control was included for safety reporting purposes and at the suggestion of the FDA given the short course of therapy and the availability of physician-selected standard-of-care for treatment failures. Finally, 11 subjects in the ITT group failed to return for test-of-eradication, with the majority lost to follow-up. Further studies will focus more intensively on subject follow-up and retention.

\section{Materials and Methods}

The study was registered at ClinicalTrials.gov as NCT01980095.

\subsection{Ethical Approval}

All procedures performed in this study involving human participants were in accordance with the ethical standards of the institutional and/or national research committee and with the 1964 Helsinki declaration and its later amendments or comparable ethical standards.

\subsection{Participant Eligibility}

Treatment-naive adults (age 18-65 years) with dyspepsia at least two weeks in duration and who had active $H$. pylori infection-based on positive ${ }^{13} \mathrm{C}$ urea breath test (UBT), confirmed by fecal antigen test or diagnosis by gastric biopsy within 12 weeks of screening or prior to randomization-were eligible for enrollment. Participants were required to use specific contraceptive methods and refrain from eating foods or taking medications known to affect CYP3A4 or CYP2C19 pathways. Participants were excluded from the study who had received prior H. pylori eradication therapy; used any antibiotics four weeks prior to screening; used any bismuth-containing medications (such as Pepto-Bismol) or PPIs within the two weeks immediately prior to screening; used prohibited medicines within s days of 
randomization; presented with $\geq$ three active gastric and/or duodenal ulcers, gastric outlet obstruction; had a history of esophageal or gastric surgery or gastric cancer within the past five years; had a positive screen for HIV or hepatitis B or C; were pregnant, lactating or, unwilling to use specified contraception; or were hypersensitive to the study drugs.

\subsection{Study Design}

ERADICATE Hp was a Phase three, randomized, double-blind, placebo-controlled study of RHB-105 in H. pylori-infected adults. The study was conducted between November 252013 and August 24 2015. Subjects were screened for study participation at 12 sites in the United States and enrolled at nine sites.

The study consisted of a screening period (Days -42 to Day 0 ) to assess subject eligibility for enrollment. Eligible participants were randomized (2:1), based on a computer-generated randomization schedule (random blocks of six and three, respectively, by site), to RHB-105 or placebo; double-blind treatment was initiated on Day one and continued for 14 days during the double-blind treatment phase. An initial test-of-eradication visit occurred 28-35 days post completion of double-blind treatment.

Those subjects who tested positive for H. pylori at the initial test-of-eradication visit were invited to enter a standard-of-care treatment phase. At the beginning of the standard-of-care phase, blinded reviewers determined the randomized treatment assignment of the subjects. Those randomized to RHB-105 were offered endoscopy and then physician-selected, susceptibility-based anti-H. pylori treatment. Those randomized to placebo were offered physician-selected anti- $H$. $p y l o r i$ therapy without endoscopy. Treatment during the standard-of-care phase was at the discretion of the treating physician and the subject. A second test-of-eradication was performed approximately 28-35 days post completion of therapy.

\subsection{Study Drug and Concomitant Medications}

The study drug consisted of RHB-105 all-in-one capsules containing $10 \mathrm{mg}$ omeprazole, $250 \mathrm{mg}$ amoxicillin, and $12.5 \mathrm{mg}$ rifabutin (Talicia ${ }^{\circledR}$, RedHill Biopharma Ltd., Raleigh, NC, USA). A matching placebo capsule was used as reference therapy. Subjects took four capsules of study drug (RHB-105) every $8 \mathrm{~h}$ for 14 days (total daily doses of amoxicillin $=3 \mathrm{~g}$, omeprazole $=120 \mathrm{mg}$, and rifabutin $=150 \mathrm{mg}$ ).

Antibiotics, PPIs (other than that contained within the study drug), and bismuth-containing drugs were prohibited during treatment and post-treatment through the test-of-eradication visit. Histamine-2 receptor antagonists and antacids were prohibited $24 \mathrm{~h}$ prior to ${ }^{13} \mathrm{C}$ UBT testing.

\subsection{Efficacy and Safety Assessments}

Patients underwent follow-up ${ }^{13} \mathrm{C}$ UBT testing (BreathTek ${ }^{\circledR}$, Otsuka America Pharmaceutical Inc., Rockville, MD) at the test-of-eradication visit to determine H. pylori eradication. Subjects in the RHB-105 group with positive ${ }^{13} \mathrm{C}$ UBT results were offered endoscopy with culture and antibiotic susceptibility testing. Pharmacogenetic testing was performed on blood samples collected at baseline to assess CYP 2C19 status. Safety of study drug was assessed based on the occurrence and severity of adverse events during the study as well as changes from baseline in hematology, chemistry, and urinalysis laboratory values.

\subsection{Statistical Methods}

All participants who received at least one dose of randomized study drug were included in the safety analysis data set. Protocol-defined analyses of the primary endpoint were performed in a modified intent-to-treat (mITT) population, which included all subjects who received at least one dose of randomized study treatment and underwent ${ }^{13} \mathrm{C}$ UBT test-of-eradication 28-35 days post-completion of therapy. The mITT population was selected to assess actual clinically relevant treatment eradication among the study subjects. Sensitivity analyses were performed in an intent-to-treat (ITT) population, 
in which subjects who were lost to treatment, dropped out of the study, or failed to return for test-of-eradication were imputed as treatment failures. Sensitivity analyses were also performed in a per-protocol (PP) population, which included all subjects who consumed at least 75\% of planned study treatment received, underwent ${ }^{13} \mathrm{C}$ UBT at the initial test-of-eradication visit, and did not have any major protocol violations.

\subsubsection{Efficacy Analysis}

The primary endpoint was $H$. pylori eradication rate at the initial test-of-eradication visit, assessed in the mITT population. The H. pylori eradication rate among the RHB-105 subjects was compared to an estimated literature-derived comparator $H$. pylori eradication rate of 70\% [27-29] using a one-sample Z-test. Sensitivity analysis by center was performed for the mITT population, with sites that enrolled four or fewer subjects combined for the analysis. Sensitivity analyses on the primary endpoint in the ITT and PP populations were also performed using a one-sample Z-test. A similar analysis was provided for the mITT and ITT population, assessing the eradication rates of physician-selected standard-of-care.

\subsubsection{Exploratory Efficacy Analyses}

Due to the fact that omeprazole is metabolized by CYP 2C19, and rifabutin is a CYP inducer, pharmacogenetic testing was performed on blood samples collected at baseline to assess CYP 2C19 status. The primary endpoint was analyzed by CYP 2C19 genotype and phenotype (classified as ultra-rapid, rapid, normal, intermediate, and poor metabolizers). In addition, culture and sensitivity testing to rifabutin, amoxicillin, clarithromycin, and metronidazole was offered for all subjects randomized to RHB-105 who were treatment failures at the initial test-of-eradication visit $(n=7)$. Most $(n=4)$ chose not to have endoscopy; among the few that did $(n=3)$, results were generally not yet available to treating physicians prior to study completion and were generally not pursued by investigators or subjects. The collected samples were analyzed using a validated methodology and reported separately.

\subsection{Data Sharing Statement}

The study protocol, statistical analysis plan, informed consent form, and data collected for this study, including de-identified individual participant data and a data dictionary defining each field in the set, will be made available upon consideration and approval of RedHill Biopharma following the publication of this paper.

\section{Conclusions}

This study showed that RHB-105 provides both efficacious and well-tolerated treatment for the eradication of $H$. pylori infection. The use of an all-in-one rifabutin-containing triple therapy provides an option for overcoming current H. pylori treatment resistance and supports the use of rifabutin-containing triple therapy as an empiric first-line treatment.

Author Contributions: D.Y.G. and I.N.K. were involved in study design. I.N.K. was involved in the overall implementation and oversight of the clinical trial. Both D.Y.G. and I.N.K. were involved with data analysis. R.M.P. was responsible for review of the study data and development of the manuscript. I.N.K., D.Y.G., D.S.R., and R.M.P. were involved in the summary of the clinical findings and finalization of the manuscript. All authors meet the International Committee of Medical Journal Editors (ICMJE) criteria, and all those who fulfilled those criteria are listed as authors. All authors have read and agreed to the published version of the manuscript.

Funding: This study was funded by RedHill Biopharma Ltd., Tel-Aviv, Israel.

Acknowledgments: Authors would like to acknowledge the subjects and investigators for their participation and efforts in the ERADICATE Hp Study. We also acknowledge Michael DeMicco (deceased) for his contribution and participation in this study. We also acknowledge the following study investigators who participated in this clinical study: California: Michael DeMicco (deceased)/Dennis S. Riff, Gilbert Martinez, Peter Winkle; Florida: Rajendra Bansal/Michael Schwartz; Georgia: Michael Steinbook; Louisiana: Robert Jeanfreau; Maryland: Robert Hardi, Michael Epstein,; Michigan: John Colombo; Nevada: Stephen Miller; North Carolina: Charles Barish, Lenin Peters. 
Conflicts of Interest: D.Y.G. is a consultant for RedHill Biopharma and Phathom Pharmaceuticals regarding novel $H$. pylori therapies, has received research support for the culturing of $H$. pylori, and is the principal investigator of an international study of the use of antimycobacterial therapy for Crohn's disease. I.N.K. was the Medical Director for this study and remains a consultant to RedHill Biopharma; I.N.K. holds company equity. D.S.R. is a key investigator in the clinical trial. R.M.P. was an employee of RedHill Biopharma at the time ERADICATE Hp was conducted and holds company equity. Employees of the Sponsor, as noted in Author Contributions, were involved in study design, data collection and analysis, and interpretation and/or other aspects pertinent to the ERADICATE Hp study, writing and/or revising the manuscript, and the decision to submit for publication.

\section{References}

1. Malfertheiner, P.; Megraud, F.; O’Morain, C.A.; Gisbert, J.P.; Kuipers, E.J.; Axon, A.T.; Bazzoli, F.; Gasbarrini, A.; Atherton, J.; Graham, D.Y.; et al. Management of Helicobacter pylori infection-the Maastricht V/Florence Consensus Report. Gut 2017, 66, 6-30. [CrossRef] [PubMed]

2. Rawla, P.; Barsouk, A. Epidemiology of gastric cancer: Global trends, risk factors and prevention. Prz. Gastroenterol. 2019, 14, 26-38. [CrossRef] [PubMed]

3. IARC Working. Group on the Evaluation of Carcinogenic Risks to Humans Schistosomes, liver flukes and Helicobacter pylori. Lyon, 7-14 June 1994. IARC Monogr. Eval. Carcinog. Risks Hum. 1994, 61, 1-241.

4. Kumar, S.; Metz, D.C.; Ellenberg, S.; Kaplan, D.E.; Goldberg, D.S. Risk factors and incidence of gastric cancer after detection of Helicobacter pylori infection: A large cohort study. Gastroenterology 2020, 158, 527-536. [CrossRef] [PubMed]

5. Barzilay, E.J.; Fagan, R.P. Chapter 3: Infectious Diseases Related to Travel. In 2012 Yellow Book-Travelers' Health-CDC; Oxford University Press Inc.: New York, NY, USA, 2011.

6. Cardenas, V.M.; Mulla, Z.D.; Ortiz, M.; Graham, D.Y. Iron deficiency and Helicobacter pylori infection in the United States. Am. J. Epidemiol. 2006, 163, 127-134. [CrossRef] [PubMed]

7. Graham, D.Y.; Adam, E.; Reddy, G.T.; Agarwal, J.P.; Agarwal, R.; Evans, D.J.; Malaty, H.M.; Evans, D.G. Seroepidemiology of Helicobacter pylori infection in India. Comparison of developing and developed countries. Dig. Dis Sci. 1991, 36, 1084-1088. [CrossRef]

8. Wannmacher, L. Review of the evidence for H. pylori treatment regimens: Section 17.1 (Review) —Adults and Children. 18th Expert Committee on the Selection and Use of Essential Medicines; World Health Organization: Geneva, Switzerland, 2011.

9. Everhart, J.E.; Kruszon-Moran, D.; Perez-Perez, G.I.; Tralka, T.S.; McQuillan, G. Seroprevalence and ethnic differences in Helicobacter pylori infection among adults in the United States. J. Infect. Dis. 2000, 181, 1359-1363. [CrossRef]

10. Dang, B.N.; Graham, D.Y. Helicobacter pylori infection and antibiotic resistance: A WHO high priority? Nat. Rev. Gastroenterol. Hepatol. 2017, 14, 383-384. [CrossRef]

11. Thung, I.; Aramin, H.; Vavinskaya, V.; Gupta, S.; Park, J.Y.; Crowe, S.E.; Valasek, M.A. Review article: The global emergence of Helicobacter pylori antibiotic resistance. Aliment. Pharmacol. Ther. 2016, 43, 514-533. [CrossRef]

12. Chey, W.D.; Leontiadis, G.I.; Howden, C.W.; Moss, S.F. ACG Clinical Guideline: Treatment of Helicobacter pylori infection. Am. J. Gastroenterol. 2017, 112, 212-239. [CrossRef]

13. Fallone, C.A.; Chiba, N.; van Zanten, S.V.; Fischbach, L.; Gisbert, J.P.; Hunt, R.H.; Jones, N.L.; Render, C.; Leontiadis, G.I.; Moayyedi, P.; et al. The Toronto consensus for the treatment of Helicobacter pylori infection in adults. Gastroenterology 2016, 151, 51-69. [CrossRef]

14. U.S. Food and Drug Administration, Health and Human Services. Establishing a list of qualifying pathogens under the Food and Drug Administration Safety and Innovation Act. Federal Register, 5 June 2014. Available online: https://www.federalregister.gov/documents/2014/06/05/2014-13023/establishing-a-list-ofqualifying-pathogens-under-the-food-and-drug-administration-safety-and-innocation-act (accessed on 5 October 2019).

15. Piccolomini, R.; Di Bonaventura, G.; Picciani, C.; Laterza, F.; Vecchiet, J.; Neri, M. In vitro activity of clarithromycin against intracellular Helicobacter pylori. Antimicrob. Agents Chemother. 2001, 45, 1568-1571. [CrossRef] [PubMed]

16. Hays, C.; Burucoa, C.; Lehours, P.; Tran, C.T.; Leleu, A.; Raymond, J. Molecular characterization of Helicobacter pylori resistance to rifamycins. Helicobacter 2018, 23. [CrossRef] [PubMed] 
17. Heep, M.; Beck, D.; Bayerdorffer, E.; Lehn, N. Rifampin and rifabutin resistance mechanism in Helicobacter pylori. Antimicrob. Agents Chemother. 1999, 43, 1497-1499. [CrossRef] [PubMed]

18. Perri, F.; Festa, V.; Andriulli, A. Treatment of antibiotic-resistant Helicobacter pylori [letter]. N. Engl. J. Med. 1998, 339, 53. [CrossRef] [PubMed]

19. Di Giulio, M.; Di Campli, E.; Di Bartolomeo, S.; Cataldi, V.; Marzio, L.; Grossi, L.; Ciccaglione, A.F.; Nostro, A.; Cellini, L. In vitro antimicrobial susceptibility of Helicobacter pylori to nine antibiotics currently used in central Italy. Scand. J. Gastroenterol. 2016, 51, 263-269. [CrossRef]

20. Biernat, M.M.; Poniewierka, E.; Błaszczuk, J.; Czapla, L.; Kempiński, R.; Ksiądzyna, D.; Grabińska, J.; Bińkowska, A.; Megraud, F.; Gościniak, G. Antimicrobial susceptibility of Helicobacter pylori isolates from lower Silesia, Poland. Arch. Med. Sci. 2014, 10, 505-509. [CrossRef]

21. Kunin, C.M. Antimicrobial activity of rifabutin. Clin. Infect. Dis. 1996, 22, S3-S13. [CrossRef]

22. Koudriakova, T.; Iatsimirskaia, E.; Tulebaev, S.; Spetie, D.; Utkin, I.; Mullet, D.; Thompson, T.; Vouros, P.; Gerber, N. In vivo disposition and metabolism by liver and enterocyte microsomes of the antitubercular drug rifabutin in rats. J. Pharmacol. Exp. Ther. 1996, 279, 1300-1309.

23. Gisbert, J.P.; Calvert, X. Review article: Rifabutin in the treatment of refractory Helicobacter pylori infection. Aliment. Pharmacol. Ther. 2012, 35, 209-221. [CrossRef]

24. Alba, C.; Blanco, A.; Alarcón, T. Antibiotic resistance in Helicobacter pylori. Curr. Opin. Infect. Dis. 2017, 30, 489-497. [CrossRef] [PubMed]

25. Graham, D.Y. Antibiotic resistance in Helicobacter pylori: Implications for therapy. Gastroenterology 1998, 115, 1272-1277. [CrossRef]

26. Borody, T.J.; Pang, G.; Wettstein, A.R.; Clancy, R.; Herdman, K.; Surace, R.; Llorente, R.; Ng, C. Efficacy and safety of rifabutin-containing 'rescue therapy' for resistant Helicobacter pylori infection. Aliment. Pharmacol. Ther. 2006, 23, 481-488. [CrossRef] [PubMed]

27. Malfertheiner, P.; Megraud, F.; O’Morain, C.A.; Atherton, J.; Axon, A.T.; Bazzoli, F.; Gensini, G.F.; Gisbert, J.P.; Graham, D.Y.; Rokkas, T.; et al. Management of Helicobacter pylori infection-The Maastricht IV/Florence Consensus Report. Gut 2012, 61, 646-664. [CrossRef] [PubMed]

28. O'Connor, A.; Gisbert, J.P.; O'Morain, C.O.; Ladas, S. Treatment of Helicobacter pylori infection 2015. Helicobacter 2015, 20, 54-61. [CrossRef] [PubMed]

29. Venerito, M.; Krieger, T.; Ecker, T.; Leandro, G.; Malfertheiner, P. Meta-analysis of bismuth quadruple therapy versus clarithromycin triple therapy for empiric primary treatment of Helicobacter pylori infection. Digestion 2013, 88, 33-45. [CrossRef] [PubMed]

30. Graham, D.Y.; Canaan, Y.; Maher, J.; Wiener, G.; Hulten, K.G.; Kalfus, I.N. Rifabutin-based triple therapy (RHB-105) for Helicobacter pylori eradication: A double-blind, randomized, controlled trial. Ann. Intern. Med. 2020, 172, 795-802. [CrossRef] [PubMed]

(C) 2020 by the authors. Licensee MDPI, Basel, Switzerland. This article is an open access article distributed under the terms and conditions of the Creative Commons Attribution (CC BY) license (http://creativecommons.org/licenses/by/4.0/). 